\title{
A formula for accelerating the convergence of a general series
}

\section{J.E. Drummond}

A weighted average of the partial sums of a series provides a quick and moderately powerful sum for any series in which the ratio of successive terms varies slowly along the series and this ratio is not close to +1 . Some properties of the sum are examined.

Let $u_{n}$ be the $n$-th term of a series and $S_{n}$ given by

$$
S_{n}=u_{1}+u_{2}+\ldots+u_{n}
$$

be a partial sum of $n$ terms of an Infinite series, $S$. Aitken's [1] two term formula for the sum may be written

$$
T_{p, p+1}=\left(S_{p+1} / u_{p+1}-S_{r} / u_{p}\right) /\left(1 / u_{r+1}-1 / u_{p}\right)
$$

and Lubkin's [2] three term formula may be written

$$
T_{r, p+2}=\left(S_{p+2} / u_{p+2}-2 S_{p+1} / u_{p+1}+S_{r} / u_{r}\right) /\left(1 / u_{r+2}-2 / u_{p+1}+1 / u_{p}\right) \text {. }
$$

We now consider a generalization of these formulae,

$$
T_{r s}=\sum_{i=0}^{s-r} w_{i} S_{r+i} / \sum_{i=0}^{s-r} w_{i} \text { where } w_{i}=(-)^{i} C_{i}^{s-r} / u_{r^{r+i}}
$$

and $c_{i}^{k}$ is the binomial coefficient $k ! / i !(k-i) !$.

$T_{r s}$ is a weighted average of the partial sums of the series and may

Received 26 August 1971. Submitted from Applied Mathematics Division, Department of Scientific and Industrial Research, Wellington, New Zealand. 
be compared with the Euler sum of a slowly convergent alternating series, $S_{r}+\frac{1}{2} u_{r+1}+\frac{1}{4}\left(u_{r+1}^{-u_{r+2}}\right)+\ldots+$ (to $k$ terms $)=\sum_{i=0}^{k} c_{i}^{k} S_{r+i} / 2^{k}$.

This is a good approximation when the terms alternate and vary slowly in size.

An approximation to the residual $\left(S-T_{r s}\right):$ Let $T_{r s}=S-E_{r s}$. If $S_{i}$ is a convergent sequence, then the truncation error $\left(S-S_{i}\right)$ tends to zero as $i \rightarrow \infty$ and $T_{r s}$ is a weighted mean of the $S_{i}$, so $E_{r s}$ also tends to zero as $r$ and $s \rightarrow \infty$ provided that the sum of the weights has a non-zero limit.

Let $u_{n+1} / u_{n}=R_{n} ;$ then

(2) $T_{r s}=s_{r-1}+u_{p} \frac{\sum_{i=0}^{s-r}(-)^{i} C_{i}^{s-r}\left(R_{s-i} R_{s-i+1} \ldots R_{s-1}\right) \sum_{j=0}^{s-i}\left(R_{r} R_{p+1} \ldots R_{s-1+j}\right)}{\sum_{i=0}^{s-r}(-)^{i} c_{i}^{s-r}\left(R_{s-i} R_{s-i+1} \cdots R_{s-1}\right)}$.

For a rapidly convergent series (that is all the $\left|R_{n}\right|<1$ )

(3) $T_{r+1, s+1}-T_{r s}=E_{r s}-E_{r+1, s+1} \doteqdot u_{s+1} \sum_{i=0}^{s-r}(-)^{i} C_{i}^{s-r_{R}}{ }_{s-i} / R_{s}$,

on picking out the largest term in the difference. We may conclude from this formula that if the ratio of terms, $R_{n}$, varies slowly with $n$ then the $(s-r)$-th order finite difference of $R_{n}$ will be small compared with $R_{n}$. Hence the first finite difference of $E_{r s}$ will be small compared with $u_{s+1}$. If, furthermore, $u_{s}$ decreases rapidly as $s$ increases, so also will $E_{r s}$ decrease as $s$ increases with $(s-r)$ constant, so we can neglect $E_{r+1, s+1}$ in our approximation and equation (2) gives an estimate of $E_{r S}$. Similarly for a rapidly divergent series (that is all the $\left|R_{n}\right|>1$ ) on picking out the largest term in the difference, 


$$
E_{r s}-E_{r+1, s+1} \doteqdot u_{r} R_{r} \sum_{i=0}^{s-r}(-)^{i} C_{i}^{s-r} / R_{r+i} .
$$

In both cases we compare the residual in the formula with the smallest term of the original series used in the weighting. If the divergent series has a meaningful sum, then the residual in the formula will be small if the $(s-r)$-th finite difference of the reciprocal of the $R_{n}$ is small compared with the reciprocal of $R_{n}$. If the transformation makes the series less divergent, $E_{r+1, s+1}$ will be larger than $E_{r s}$ while $E_{r s}$ will be the larger if the divergent series is converted to a convergent series, but in either case $E_{r s}$ will be comparable with the expression (4).

In operating on a slowly convergent or divergent series the expression for the residual is more complicated than (3) or (4) but for a slowly convergent alternating series, formula (1) approximates to the Euler sum which is known to be a good approximation for such a series. However, if $u_{p}$ is the reciprocal of a polynomial in $n$, then in equation (1), $\sum w_{i}$ is the finite difference of a polynomial, so will be zero when $(s-r)$ is sufficiently large. In this case $T_{r s}$ may be unbounded. Lubkin's formula which is $T_{r, r+2}$ fails when $u_{n}$ is the reciprocal of a linear function of $n$, but this is no loss because $\sum n^{-1}$ is itself a divergent-series. For a slowly convergent series of positive terms formula (1) is found to be unsatisfactory because the sum of the weights is small, but it works well on many other series.

As an example we sum the series $\sum(-)^{n} n !$ to show the simplicity and power of the sum. We tabulate $(10) ! / u_{n}$ and $(10) ! S_{n} / u_{n}$; then we may introduce the binomial coefficients and calculate any. $T_{r s}$ as desired. The set $T_{0 n}$ is listed in the third column of the table. Any one of these may be computed as desired and provides a relatively simple and moderately powerful method for summing a series. If greater accuracy is desired, it may be observed that the $T_{0 n}$ column appears to be a convergent sequence, so formula (1) may be applied again to this sequence. In this case the 
labour involved becomes more comparable with that of the other more powerful methods such as repeated use of Aitken's transform [1], Shanks' $e_{n}$ processes [4], Wynn's $E$ and $\rho$ algorithms [5, 6] and Rutishauser's Q-D algorithm [3]. Euler's transform is useless on this series.

TABLE. ESTIMATES OF $\sum(-)^{n} n$ !

$\begin{array}{rrc}(10) ! / n ! & (10) ! S_{n} / n ! & T_{0 n} \\ 3628800 & 3628800 & \\ 3628800 & 0 & .50000 \\ 1814400 & 3628800 & .57143 \\ 604800 & -2419200 & .58824 \\ 151200 & 3024000 & .59330 \\ 30240 & -3024000 & .59508 \\ 5040 & 3124800 & .59578 \\ 720 & -3182400 & .59608 \\ 90 & 3231000 & .59621 \\ 10 & -3269800 & .59628 \\ 1 & 331820 & .59631\end{array}$

As a second example we sum the series

$$
\pi=4-4 / 3+4 / 5+\ldots .
$$

In this example $R_{n}=(2 n-1) /(2 n+1) \cdot R_{n}$ changes rapidly with $n$ when $n$ is small, so any finite difference of $R_{n}$ or $R_{n}^{-1}$ which includes terms with $n$ near zero is not small. In this case we can do better by taking a lower order finite difference further along the series. If we use 16 terms it is best to calculate $T_{6,16}$; then we get an error of 1 in the 10-th digit. This series is also particularly good for Euler's formula since $R_{n}$ is close to 1 . However we lose strength if we include the first terms where $R_{n}$ is not close to 1 . On applying Euler's formula to the latter 10 or 11 of 16 terms, we get an error of 2 in the 9-th digit. Aitken's formula develops the same weakness when $R_{n}$ is changing 
rapidly. With somewhat more labour and six repetitions of Aitken's transform we use up 12 terms of the series before Aitken's transform becomes unstable at the start of the series. At this stage the error is in the 14-th digit. Aitken's transform may be repeated but each repetition after the sixth uses up three terms because of the initial instability so the convergence is less rapid. Aitken's method however remains the best of these three methods per number of terms used if the amount of effort used is not an important restriction.

If we tabulate $T_{1 s}$ as $s$ runs from 2 to 16 we obtain a convergent sequence of estimates of $\pi$ using equation $l$ and if desired we may repeat equation 1 on $T_{1 s}$ to get a better estimate of the sum. Alternatively, we may tabulate $T_{r, 16}$ as $r$ runs from 1 to 15 and this time we obtain an asymptotic sequence with a minimum at $r=6$ mentioned above. Equation 1 may be repeated on this sequence to get a better estimate.

In conclusion, equation ( 1 ) is a weighted Euler sum which may be used when a suitable Euler weight is unknown.

\section{References}

[1] A.C. Aitken, "On Bernoulli's numerical solution of algebraic equations", Proc. Roy. Soc. Edinburgh 46 (1925-26), 289-305.

[2] Samuel Lubkin, "A method of summing infinite series", J. Res. Nat. Bur. Standards 48 (1952), 228-254.

[3] Heinz Rutishauser, "Der Quotienten-Differenzen-Algorithmus", Mitt. Inst. angew. Math. Zürich 7. (Birkhäuser Verlag, Basel, Stuttgart, 1957).

[4] Daniel Shanks, "Non-linear transformations of divergent and slowly convergent sequences", J. Math. and Phys. 34 (1955), 1-42.

[5] P. Wynn, "On a device for computing the $e_{m}\left(s_{n}\right)$ transformation", Math. Tables Aids Comput. 10 (1956), 91-96. 
[6] P. Wynn, "On a procrustean technique for the numerical transformation of slowly convergent sequences and series", Proc. Combridge Phizos. Soc. 52 (1956), 663-671.

Department of Applied Mathematics, School of General Studies, Australian National University, Canberra, ACT. 